\title{
Testis-specific Y-centric protein-protein interaction network provides clues to the etiology of severe spermatogenic failure
}

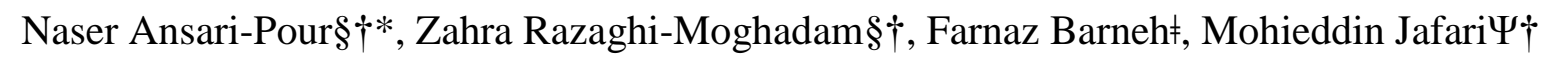

$\S$ Faculty of New Sciences and Technology, University of Tehran, Tehran, Iran.

‡ Faculty of Paramedical Sciences, Shahid Beheshti University of Medical Sciences, Tehran, Iran

$\Psi$ Drug Design and Bioinformatics Unit, Medical Biotechnology Department, Biotechnology Research Center, Pasteur Institute of Iran, Tehran, Iran.

$\uparrow$ School of Biological Sciences, Institute for Research in Fundamental Sciences (IPM), Tehran, Iran.

*Corresponding author: Dr Naser Ansari-Pour, Email: n.ansaripour@ut.ac.ir, Tel: +98-2161118438

\section{Supplementary Information:}

Figure S-1

Tables S-1 to S-8

Note: Table S-8 is provided separately in an accompanying Excel file due to its length. 
Figure S-1. Principal component analysis (PCA) plot of SpF and control samples based on the expression profile of the $\mathrm{Y}$ chromosome.

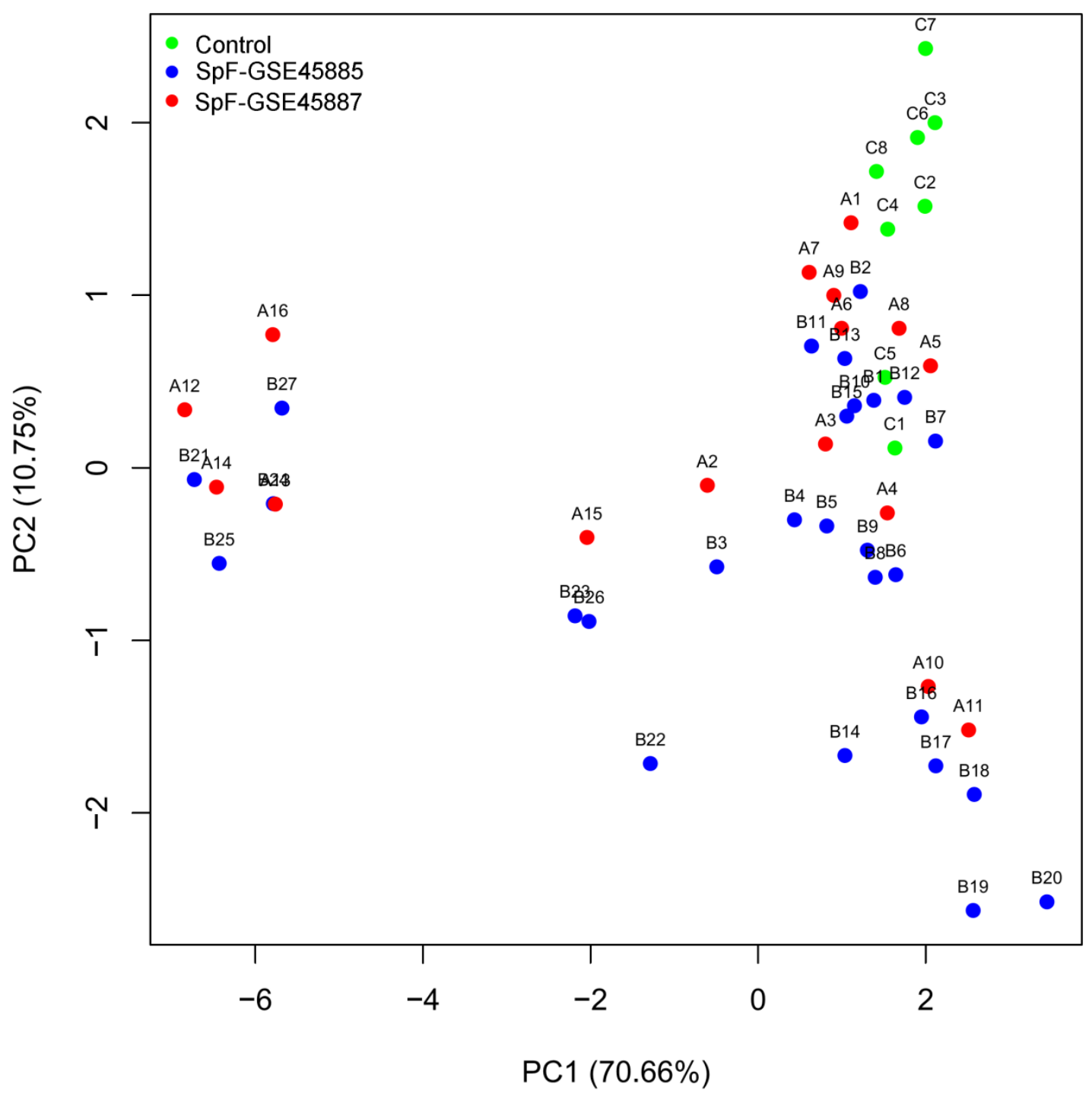


Table S-1. Topological parameter values of genes in the Y co-expression network.

\begin{tabular}{|l|c|c|c|}
\hline Y gene & Degree & Betweenness centrality & Clustering coefficient \\
\hline CDY1 & 3 & 0 & 0.295 \\
\hline DAZ1 & 6 & 0.092 & 0.439 \\
\hline DDX3Y & 5 & 0.425 & 0.474 \\
\hline EIF1AY & 4 & 0.366 & 0.383 \\
\hline HSFY1 & 6 & 0.019 & 0.429 \\
\hline KDM5D & 5 & 0.140 & 0.409 \\
\hline NLGN4Y & 7 & 0.264 & 0.450 \\
\hline PCDH11Y & 6 & 0.101 & 0.486 \\
\hline RBMY1A1 & 6 & 0.101 & 0.486 \\
\hline RPS4Y1 & 3 & 0.018 & 0.353 \\
\hline RPS4Y2 & 1 & 0 & 0.254 \\
\hline SRY & 1 & 0 & 0.234 \\
\hline TBL1Y & 1 & 0 & 0.295 \\
\hline TMSB4Y & 3 & 0.111 & 0.333 \\
\hline TSPY1 & 4 & 0.111 & 0.300 \\
\hline USP9Y & 7 & 0.142 & 0.486 \\
\hline UTY & 6 & 0.074 & 0.462 \\
\hline XKRY & 3 & 0.015 & 0.360 \\
\hline ZFY & 3 & 0 & 0.295 \\
\hline & & &
\end{tabular}


Table S-2. Details of Y genes and their differential expression profile.

\begin{tabular}{|c|c|c|c|c|c|c|c|}
\hline $\begin{array}{l}\text { Gene } \\
\text { symbol* }\end{array}$ & Gene name (description) & Chr band & $\begin{array}{l}\text { Gene start } \\
\text { (bp) }\end{array}$ & Gene end (bp) & $\begin{array}{l}\text { AZF } \\
\text { region }\end{array}$ & $\begin{array}{l}\text { Expression } \\
\text { change } \\
(\mathrm{P}(\text { corrected }))\end{array}$ & $\begin{array}{l}\text { Fold } \\
\text { change }\end{array}$ \\
\hline AMELY & amelogenin, Y-linked & Yp11.2 & 6865918 & 6874027 & - & 1 & 1.05 \\
\hline BPY2 & basic charge, Y-linked, 2 & Yq11.223 & 22973819 & 23005465 & $\mathrm{AZFC}$ & 0.00031 & 0.65 \\
\hline CDY1 & chromodomain protein, Y-linked, 1 & Yq11.23 & 25622162 & 25624902 & $\mathrm{AZFC}$ & 0.055 & 0.50 \\
\hline DAZ1 & deleted in azoospermia 1 & Yq11.223 & 23129355 & 23199094 & $\mathrm{AZFC}$ & $1.30 \mathrm{E}-11$ & 0.04 \\
\hline DDX3Y & DEAD (Asp-Glu-Ala-Asp) box helicase 3, Y-linked & Yq11.221 & 12904108 & 12920478 & AZFa & 1 & 0.90 \\
\hline EIF1AY & eukaryotic translation initiation factor $1 \mathrm{~A}, \mathrm{Y}$-linked & Yq11.223 & 20575725 & 20593154 & $\mathrm{AZFb}$ & 0.0022 & 1.92 \\
\hline HSFY1 & heat shock transcription factor, Y-linked 1 & Yq11.222 & 18546671 & 18588963 & $\mathrm{AZFb}$ & 0.00027 & 0.66 \\
\hline KDM5D & lysine $(\mathrm{K})$-specific demethylase 5D & Yq11.223 & 19703865 & 19744939 & $\mathrm{AZFb}$ & 0.026 & 1.44 \\
\hline NLGN4Y & neuroligin 4, Y-linked & Yq11.221 & 14522638 & 14845650 & - & 0.0024 & 2.68 \\
\hline PCDH11Y & protocadherin $11 \mathrm{Y}$-linked & Yp11.2 & 5000226 & 5742224 & - & 1 & 1.41 \\
\hline PRY & PTPN13-like, Y-linked & Yq11.223 & 22490397 & 22514637 & AZFc & 1 & 1.17 \\
\hline RBMY1A1 & RNA binding motif protein, Y-linked, family 1, member A1 & Yq11.223 & 21511372 & 21549326 & $\mathrm{AZFb}$ & $6.80 \mathrm{E}-07$ & 0.25 \\
\hline RPS4Y1 & ribosomal protein S4, Y-linked 1 & Yp11.2 & 2841486 & 2932000 & - & $7.15 \mathrm{E}-06$ & 1.86 \\
\hline RPS4Y2 & ribosomal protein S4, Y-linked 2 & Yq11.223 & 20756164 & 20781032 & $\mathrm{AZFb}$ & 0.00024 & 1.32 \\
\hline SRY & sex determining region $Y$ & Yp11.2 & 2786855 & 2787699 & - & 1 & 0.95 \\
\hline TBL1Y & transducin (beta)-like 1, Y-linked & Yp11.2 & 6910686 & 7091683 & - & 0.085 & 1.13 \\
\hline TGIF2LY & TGFB-induced factor homeobox 2-like, Y-linked & Yp11.2 & 3579041 & 3580041 & - & $1.80 \mathrm{E}-06$ & 0.25 \\
\hline TMSB4Y & thymosin beta 4, Y-linked & Yq11.221 & 13703567 & 13706024 & - & 0.00013 & 2.00 \\
\hline TSPY1 & testis specific protein, Y-linked 1 & Yp11.2 & 9466955 & 9490081 & - & $2.80 \mathrm{E}-09$ & 0.07 \\
\hline USP9Y & ubiquitin specific peptidase $9, \mathrm{Y}$-linked & Yq11.221 & 12701231 & 12860839 & $\mathrm{AZFa}$ & 0.00033 & 2.89 \\
\hline UTY & ubiquitously transcribed tetratricopeptide repeat containing, Y-linked & Yq11.221 & 13248379 & 13480673 & - & 0.0077 & 3.15 \\
\hline VCY & variable charge, Y-linked & Yq11.221 & 13985772 & 13986513 & - & $1.80 \mathrm{E}-07$ & 0.23 \\
\hline XKRY & XK, Kell blood group complex subunit-related, Y-linked & Yq11.222 & 17768980 & 17777400 & - & 0.55 & 3.10 \\
\hline ZFY & zinc finger protein, Y-linked & Yp11.2 & 2935281 & 2982506 & - & 0.14 & 1.26 \\
\hline
\end{tabular}

*Differentially expressed genes are shown in bold type. 
Table S-3. List of all common immediate interactors of X-degenerate Y proteins.

\begin{tabular}{|c|c|c|c|c|}
\hline MRPS10 & ENSP00000454786 & NOP14 & RPL30 & RPS24 \\
\hline ANKRD42 & ENSP00000463847 & NOP56 & RPL31 & RPS25 \\
\hline BMS1 & FAU & NOP58 & RPL32 & RPS26 \\
\hline BYSL & FBL & NPSR1 & RPL34 & RPS27 \\
\hline C9orf114 & GMPS & NSA2 & RPL35 & RPS27A \\
\hline CAD* & GNB2L1 & OAS1 & RPL35A & RPS27L \\
\hline ССТ5 & HDAC3 & OAS2 & RPL36 & RPS28 \\
\hline CPS1 & IMP3 & OAS3 & RPL36A & RPS29 \\
\hline DDX24 & IMPDH2 & OASL & RPL36AL & RPS3 \\
\hline DENR & ISG15 & PDCD11 & RPL37 & RPS3A \\
\hline DKC1 & KIAA0020 & PLEC & RPL37A & RPS4X \\
\hline EEF1A1 & KRI1 & PNO1 & RPL38 & RPS5 \\
\hline EEF1A2 & KRR1 & PSMD10 & RPL39 & RPS6 \\
\hline EEF1B2 & MRPL1 & PWP2 & RPL39L & RPS7 \\
\hline EEF1D & MRPL11 & RAD23A & RPL3L & RPS8 \\
\hline EEF1G & MRPL13 & RAD23B & RPL4 & RPS9 \\
\hline EEF2 & MRPL15 & $\mathrm{RCL} 1$ & RPL5 & RPSA \\
\hline EFTUD2 & MRPL16 & RPL10 & RPL6 & RPSAP58 \\
\hline EIF1 & MRPL2 & RPL10A & RPL7 & RRP9 \\
\hline EIF1B & MRPL22 & RPL10L & RPL7A & RSL24D1 \\
\hline EIF2S2 & MRPL24 & RPL11 & RPL7L1 & RSRC1 \\
\hline EIF3B & MRPL3 & RPL12 & RPL8 & SEC61A1 \\
\hline EIF3C & MRPL4 & RPL13 & RPL9 & SEC61A2 \\
\hline EIF3F & MRPS11 & RPL13A & RPLPO & SETD1A \\
\hline EIF3G & MRPS12 & RPL14 & RPLP1 & SETD1B \\
\hline EIF3H & MRPS14 & RPL15 & RPLP2 & TPT1 \\
\hline EIF3I & MRPS15 & RPL17 & RPP38 & TRUB1 \\
\hline EIF3M & MRPS2 & RPL18 & RPS10 & TSR1 \\
\hline EIF4A1 & MRPS5 & RPL18A & RPS11 & UBA52 \\
\hline EIF4A2 & MRPS7 & RPL19 & RPS12 & UBB \\
\hline EIF4A3 & MRPS9 & RPL21 & RPS13 & UBBP4 \\
\hline EIF4E & MRTO4 & RPL22 & RPS14 & UBC \\
\hline EIF4G1 & NACA & RPL22L1 & RPS15 & UBD \\
\hline
\end{tabular}




\begin{tabular}{|l|l|l|l|l|} 
EIF5 & NACA2 & RPL23 & RPS15A & UBL4A \\
\hline EIF5A & NACAD & RPL23A & RPS16 & UBL4B \\
\hline EIF5A2 & NAT10 & RPL24 & RPS17 & UBQLN1 \\
\hline EIF5AL1 & NCBP1 & RPL26 & RPS17L & UBQLN2 \\
\hline EIF5B & NCBP2 & RPL26L1 & RPS18 & UBQLN4 \\
\hline ENSP00000310146 & NEDD8 & RPL27 & RPS19 & UBQLNL \\
\hline ENSP00000386655 & NHP2 & RPL27A & RPS2 & UTP15 \\
\hline ENSP00000389465 & NHP2L1 & RPL28 & RPS20 & UTP6 \\
\hline ENSP00000403936 & NOC4L & RPL29 & RPS21 & WDR31 \\
\hline ENSP00000415634 & NOL6 & RPL3 & RPS23 & ZFAND4 \\
\hline
\end{tabular}

*Interactors shared with X-linked homologues are shown in bold type. 
Table S-4. Expression change profile of SpF-implicated genes (SIGs).

\begin{tabular}{|c|c|c|}
\hline Gene & $\begin{array}{l}\text { Expression change } \\
\text { (P(corrected)) }\end{array}$ & Fold change \\
\hline ANKS1B & 1 & 0.79 \\
\hline AVEN & 1 & 0.91 \\
\hline DDX11* & 0.00053 & 0.48 \\
\hline EPHA3 & 1 & 1.20 \\
\hline ESR1 & 1 & 1.40 \\
\hline ESR2 & 0.009 & 0.36 \\
\hline FASLG & 0.1 & 1.29 \\
\hline HSD17B4 & 0.0022 & 2.07 \\
\hline KIAA1267 & 0.0011 & 0.69 \\
\hline LAMB2 & 0.63 & 1.44 \\
\hline MMEL1 & 0.049 & 0.38 \\
\hline NANOS1 & 1 & 1.06 \\
\hline NR5A1 & 1 & 0.40 \\
\hline PEX10 & 0.23 & 0.70 \\
\hline PGR & 0.78 & 1.98 \\
\hline PLEC & 1 & 1.36 \\
\hline PRMT6 & 1 & 1.28 \\
\hline SIRPA & 1 & 1.16 \\
\hline SIRPG & 1 & 0.90 \\
\hline SOX5 & 1 & 1.22 \\
\hline SYCP3 & $1.10 \mathrm{E}-06$ & 0.20 \\
\hline TAF4B & 0.077 & 0.41 \\
\hline UGT2B17 & 1 & 0.95 \\
\hline ZMYND15 & 0.00047 & 0.19 \\
\hline
\end{tabular}

*Differentially expressed genes are shown in bold type. 
Table S-5. Expression change profile of ESR2 and AR downstream genes (TRED).

\begin{tabular}{|c|c|c|c|}
\hline $\begin{array}{l}\text { Transcription } \\
\text { factor }\end{array}$ & Downstream gene & $\begin{array}{l}\text { Expression change } \\
(\mathrm{P}(\text { corrected }))\end{array}$ & $\begin{array}{l}\text { Fold } \\
\text { change }\end{array}$ \\
\hline \multirow{27}{*}{ ESR2 } & $\mathrm{C} 3$ & 1 & 1.36 \\
\hline & CD68* & 0.0092 & 2.61 \\
\hline & CREBBP & 0.004 & 0.75 \\
\hline & CRHBP & 1 & 1.22 \\
\hline & CRYZ & 0.00068 & 7.64 \\
\hline & CTSD & 0.13 & 1.34 \\
\hline & EBAG9 & 0.1 & 1.46 \\
\hline & EGFR & $6.50 \mathrm{E}-06$ & 2.24 \\
\hline & GRIN2D & 1 & 0.97 \\
\hline & JUN & 1 & 1.50 \\
\hline & MAD2L1 & 0.15 & 0.75 \\
\hline & MAOB & 0.42 & 2.60 \\
\hline & MKNK2 & 1 & 0.90 \\
\hline & NCOA3 & 1 & 1.11 \\
\hline & NOS3 & 1 & 0.95 \\
\hline & NQO1 & 0.001 & 3.08 \\
\hline & PGR & 0.87 & 1.98 \\
\hline & PNRC1 & 0.0000025 & 1.62 \\
\hline & POU1F1 & 1 & 1.12 \\
\hline & PPARG & 0.98 & 0.48 \\
\hline & PTPRC & 0.000011 & 2.03 \\
\hline & SELE & 1 & 0.97 \\
\hline & SERPINE1 & 1 & 1.00 \\
\hline & SMARCA1 & 0.11 & 2.12 \\
\hline & SMARCA2 & 1 & 1.11 \\
\hline & STAT5A & 0.35 & 1.40 \\
\hline & TFF1 & 0.29 & 1.27 \\
\hline \multirow{11}{*}{ AR } & ACPP & 0.73 & 2.32 \\
\hline & CCNG1 & 0.000028 & 3.87 \\
\hline & CDKN1A & 1 & 0.77 \\
\hline & ECM1 & 0.26 & 7.30 \\
\hline & ELK1 & 1 & 1.16 \\
\hline & FGF2 & 0.013 & 1.93 \\
\hline & FGFBP1 & 1 & 1.15 \\
\hline & FHL2 & 1 & 0.89 \\
\hline & FOXA1 & 0.019 & 0.71 \\
\hline & GTF2F1 & 0.003 & 0.56 \\
\hline & H3F3A & 0.072 & 1.27 \\
\hline
\end{tabular}




\begin{tabular}{|l|l|r|r|}
\hline IGFBP5 & 0.72 & 2.47 \\
\cline { 2 - 4 } IL6 & 0.053 & 1.19 \\
\cline { 2 - 4 } IL6ST & 0.00071 & 3.19 \\
\hline KLK14 & 1 & 1.10 \\
\hline LCP1 & 1 & 2.63 \\
\hline MYC & 1 & 0.75 \\
\hline NCOA1 & 1 & 0.99 \\
\hline PGC & 1 & 0.98 \\
\cline { 2 - 4 } & PIGR & 0.65 & 1.34 \\
\cline { 2 - 4 } & PLAU & 0.016 & 2.12 \\
\hline & PNRC1 & 0.0000021 & 1.62 \\
\cline { 2 - 4 } & UBE3A & 1 & 1.24 \\
\hline
\end{tabular}

*Differentially expressed genes are shown in bold type. 
Table S-6. Expression change profile of murine SCOS genes (mSCOS).

\begin{tabular}{|l|r|r|}
\hline Gene & Expression change (P(corrected)) & Fold change \\
\hline ACVR2A & 0.18 & 1.58 \\
\hline AR & $5.20 E-07$ & 2.30 \\
\hline B4GALNT1 & 1 & 1.19 \\
\hline BCL2L2 & 0.2 & 1.44 \\
\hline CDKN2C & 1 & 1.02 \\
\hline CLDN11 & 0.36 & 0.23 \\
\hline CYP17A1 & 1 & 0.18 \\
\hline CYP19A1 & 1 & 1.06 \\
\hline DHH & 1 & 0.39 \\
\hline DMRT1 & 0.19 & 0.34 \\
\hline DNAJA1 & 0.0045 & 0.76 \\
\hline GDI1 & 0.47 & 0.66 \\
\hline GDNF & 1 & 1.09 \\
\hline GJA1 & 1 & 1.09 \\
\hline HMGA1 & $7.70 E-06$ & 0.38 \\
\hline HMGB2 & 0.00034 & 0.45 \\
\hline INHA & 1 & 0.33 \\
\hline KITLG & 0.025 & 2.49 \\
\hline LHCGR & 1 & 1.00 \\
\hline MAN2A2 & 0.0007 & 0.40 \\
\hline MAP7 & 1 & 0.68 \\
\hline NR0B1 & 0.36 & 0.24 \\
\hline RBP4 & 1 & 0.68 \\
\hline SBF1 & 0.00034 & 0.25 \\
\hline SERPINA5 & 1 & 0.08 \\
\hline SF1 & 1 & 0.81 \\
\hline SLC12A2 & 0.79 \\
\hline SOX8 & 0.84 \\
\hline & 1 & \\
\hline
\end{tabular}

*Differentially expressed genes are shown in bold type. 
Table S-7. SpF network-drug interaction.

\begin{tabular}{|c|c|c|c|}
\hline $\begin{array}{l}\text { Gene symbol } \\
\text { (Uniprot code) }\end{array}$ & Manipulating Drug & Drug type & $\begin{array}{l}\text { Drug mode of } \\
\text { action }\end{array}$ \\
\hline ESR1 (P03372) & $\begin{array}{l}\text { Clomifene } \\
\text { Melatonin } \\
\text { Tamoxifen }\end{array}$ & $\begin{array}{l}\text { approved, investigational } \\
\text { approved, nutraceutical } \\
\text { approved }\end{array}$ & $\begin{array}{l}\text { antagonist/agonist } \\
\text { antagonist } \\
\text { antagonist/agonist }\end{array}$ \\
\hline ESR2 (Q92731) & $\begin{array}{l}\text { Tamoxifen } \\
\text { Raloxifene } \\
\text { Trilostane } \\
\text { Genistein }\end{array}$ & $\begin{array}{l}\text { approved } \\
\text { approved, investigational } \\
\text { approved, investigational, withdrawn } \\
\text { investigational }\end{array}$ & $\begin{array}{l}\text { antagonist/agonist } \\
\text { agonist } \\
\text { allosteric } \\
\text { modulator } \\
\text { unknown }\end{array}$ \\
\hline $\begin{array}{l}\text { RPL10L } \\
(\mathrm{Q} 96 \mathrm{~L} 21)\end{array}$ & $\begin{array}{l}\text { Anisomycin } \\
\text { Puromycin }\end{array}$ & $\begin{array}{l}\text { experimental } \\
\text { experimental }\end{array}$ & $\begin{array}{l}\text { unknown } \\
\text { unknown }\end{array}$ \\
\hline RPL7 (P18124) & Bortezomib & approved, investigational & inhibitor \\
\hline RPL23 (P62829) & $\begin{array}{l}\text { Anisomycin } \\
\text { Puromycin }\end{array}$ & $\begin{array}{l}\text { experimental } \\
\text { experimental }\end{array}$ & $\begin{array}{l}\text { unknown } \\
\text { unknown }\end{array}$ \\
\hline FGF2 (P09038) & $\begin{array}{l}\text { Sucralfate } \\
\text { Pentosan Polysulfate } \\
\text { Sirolimus } \\
\end{array}$ & $\begin{array}{l}\text { approved } \\
\text { approved } \\
\text { approved, investigational }\end{array}$ & $\begin{array}{l}\text { agonist } \\
\text { antagonist } \\
\text { unknown } \\
\end{array}$ \\
\hline PLAU (P00749) & Amiloride & approved & inhibitor \\
\hline EGFR (P00533) & $\begin{array}{l}\text { Vandetanib } \\
\text { Afatinib } \\
\text { Gefitinib } \\
\text { Erlotinib } \\
\text { Lapatinib } \\
\text { Panitumumab } \\
\end{array}$ & $\begin{array}{l}\text { approved } \\
\text { approved } \\
\text { approved, investigational } \\
\text { approved, investigational } \\
\text { approved, investigational } \\
\text { approved, investigational }\end{array}$ & $\begin{array}{l}\text { inhibitor } \\
\text { inhibitor } \\
\text { antagonist } \\
\text { antagonist } \\
\text { antagonist } \\
\text { suppressor }\end{array}$ \\
\hline AR (P10275) & $\begin{array}{l}\text { Flutamide } \\
\text { Nilutamide } \\
\text { Fludrocortisone } \\
\text { Bicalutamide } \\
\text { Drospirenone } \\
\text { Danazol } \\
\text { Testosterone } \\
\text { Propionate } \\
\text { Methyltestosterone } \\
\text { Enzalutamide } \\
\text { Levonorgestrel } \\
\text { Oxandrolone } \\
\text { Testosterone } \\
\text { Ketoconazole } \\
\text { Cyproterone acetate }\end{array}$ & $\begin{array}{l}\text { approved } \\
\text { approved } \\
\text { approved } \\
\text { approved } \\
\text { approved } \\
\text { approved } \\
\text { approved } \\
\text { approved } \\
\text { approved } \\
\text { approved, investigational } \\
\text { approved, investigational } \\
\text { approved, investigational } \\
\text { approved, investigational } \\
\text { approved, investigational }\end{array}$ & $\begin{array}{l}\text { antagonist } \\
\text { antagonist } \\
\text { agonist } \\
\text { antagonist } \\
\text { antagonist } \\
\text { agonist } \\
\text { agonist } \\
\text { agonist } \\
\text { inhibitor } \\
\text { agonist } \\
\text { agonist } \\
\text { agonist } \\
\text { binder } \\
\text { antagonist }\end{array}$ \\
\hline
\end{tabular}

